\title{
Zusatzweiterbildung Phlebologie ist gerettet
}

\author{
M. Stücker'; A. Mumme²; E. Mendoza ${ }^{3} ;$ K. Hartmann"; F. Pannier ${ }^{5}$; E. Rabe'; H. Gerlach ${ }^{7}$ \\ ${ }^{1}$ Klinik für Dermatologie der Ruhr-Universität Bochum, Venenzentrum der Dermatologischen und Gefäßchirurgischen \\ Kliniken, Kliniken der Ruhr-Universität Bochum; ${ }^{2}$ Klinik für Gefäßchirurgie der Ruhr-Universität Bochum, Venenzentrum \\ der Dermatologischen und Gefäßchirurgischen Kliniken, Kliniken der Ruhr-Universität Bochum; ${ }^{3}$ Venenpraxis Wuns- \\ torf; ${ }^{4}$ Venenzentrum Freiburg; ${ }^{5}$ Klinik und Poliklinik für Dermatologie und Venerologie, Universitätsklinikum Köln; ${ }^{6} \mathrm{Kli}-$ \\ nik und Poliklinik für Dermatologie, Bonn; ${ }^{7}$ Praxis Dr. Gerlach Mannheim
}

Am Mittwoch 25. April 2018 um 16:41 Uhr wurde schriftlich das bestätigt, was Prof. Dr. Markus Stücker, amtierender Präsident der Deutschen Gesellschaft für Phlebologie, bereits auf mündliche Nachfrage telefonisch von der Bundesärztekammer erfahren hatte: die Zusatzweiterbildung Phlebologie ist vom Vorstand der Bundesärztekammer, der am Freitag 20.04.2018 getagt hatte, nicht zum Beibehalt in der MusterWeiterbildungsordnung empfohlen worden. In der Mitteilung hieß es: „Einige Zusatzweiterbildungen sind mehrheitlich nicht zur Aufnahme bzw. nicht zum Beibehalt in der (Muster-)Weiterbildungsordnung empfohlen worden [...]: Chinesische Medizin, klinische Umweltmedizin, Labordiagnostik, Lymphologie, osteopathische Medizin, Phlebologie, Reisemedizin, Röntgendiagnostik, Sexualmedizin, spezielle dermatologische Operationen, spezielle Herzchirurgie, spezielle Sozialpädiatrie, spezielle Stoffwechselmedizin im Kindesund Jugendalter, spezielle Strabologie und Neuroophthalmologie, spezielle Viszeralchirurgie." Außer der Phlebologie sollten demnach lediglich die Labordiagnostik, Röntgendiagnostik und spezielle Viszeralchirurgie als Zusatzweiterbildung abgeschafft werden.

Noch am gleichen Abend wurde von Prof. Dr. Markus Stücker, Vorsitzender der

Korrespondenzadresse

Prof. Dr. med. Markus Stücker

Klinik für Dermatologie der Ruhr-Universität Bochum

Venenzentrum der Dermatologischen und Gefäßchirurgischen Kliniken

Kliniken der Ruhr-Universität Bochum

Im St. Maria-Hilf-Krankenhaus

Hiltroper Landwehr 11-13

44805 Bochum

Tel. 0234 8792-377

E-Mail: m.stuecker@klinikum-bochum.de

www.venenzentrum-uniklinik.de

Deutschen Gesellschaft für Phlebologie, mit Dr. Horst Gerlach als dem Vorsitzenden des Berufsverbands der Phlebologen, Frau PD Felizitas Pannier als Mitglied des erweiterten Vorstands der DGP und Herrn Stefan Unger eine Telefonkonferenz abgehalten, um das weitere Vorgehen zu besprechen. Bemerkenswert war an der Situation, dass die Fachgesellschaften zwar in den Novellierungsprozess der Muster-Weiterbildungsordnung eingebunden worden waren, um Modifikationen der Weiterbildungsordnungen des jeweiligen Fachs einzubringen. So brachte auch die Deutsche Gesellschaft für Phlebologie bspw. als Ergänzung ein, die thermisch ablativen Verfahren in den Weiterbildungskatalog aufzunehmen. Eine formelle Befragung aber über die Notwendigkeit der Zusatzweiterbildung Phlebologie hat weder bei der DGP noch beim BVP, und wie sich im weiteren herausstellte auch nicht bei der Deutschen Dermatologischen Gesellschaft, dem Berufsverband der Deutschen Dermatologen, der Deutschen Gesellschaft für Gefäßchirurgie und Gefäßmedizin und auch nicht bei der Deutschen Gesellschaft für Angiologie stattgefunden.

Die einzige Chance, den Vorschlag des Vorstands der Bundesärztekammer nicht Realität werden zu lassen, lag darin, auf dem 121. Deutschen Ärztetag in Erfurt

\section{Zitierweise des Beitrags:}

Additional medical education phlebology is saved Phlebologie 2018; 47: 176-180 https://doi.org/10.12687/phleb2429-4-2018 Eingereicht: 30. Mai 2018

Angenommen: 05. Juni 2018 vom 08.-11. Mai 2018 ein entsprechendes Votum zu erreichen. Drei Schritte bzw. Möglichkeiten konnten dabei definiert werden.

1. Ordentliche Vorgehensweise: Antragstellung gemäß $\$ 11$ der Geschäftsordnung des Deutschen Ärztetages

a) Einbringungsmöglichkeiten des Antrags - i)Vor Beginn des Ärztetags: Schriftliche Antragstellung durch sämtliche stimmberechtigte Abgeordnete einer LÄK und deren Präsidenten

- ii) Nach Beginn des Ärztetags: Schriftliche Antragstellung durch zehn stimmberechtigte Abgeordnete

b) Ausarbeitungsmöglichkeiten des Antrags

- i) Antrag auf Vertagung der Abstimmung aufgrund intransparenter Informationslage

- ii)Gegenantrag zur Beibehaltung der Zusatz-Weiterbildung Phlebologie

- iii) Antrag auf Rücküberweisung an den Vorstand der BÄK

2. Außerordentliche Vorgehensweise: Schriftliche Kontaktaufnahme mit Delegierten und telefonisches Nachfassen

a) Handlungsaufforderung in Abhängigkeit von Möglichkeit der Antragstellung - i) Aufforderung zur Zustimmung zum eigenen Antrag oder

- ii) Aufforderung zur Ablehnung der MWBO-Novellierung mit Konsequenz Wegfall der Zusatz-Weiterbildung Phlebologie

b) Argumentative Grundlagen

- i) Intransparente Informationslage und mangelnde Möglichkeiten zur Stellungnahme

- ii) Bedeutung der Zusatz-Weiterbildung Phlebologie für die Gesundheitsversorgung

c) Nutzung eigener Netzwerke 
d) Nach Möglichkeit Allianzbildung mit DGG und DDG

e) Ggf. „Brandbrief“ an BÄK-Vorstand

3. Außerordentliche Vorgehensweise: Öffentlichkeitswirksame Maßnahmen unter Einschaltung von Patientenvertretern \& ggf. Politik

a) Aufsetzen einer PR-Kampagne in Fachund ggf. Publikumsmedien, Versand einer Pressemitteilung etc.

b) Kontaktaufnahme mit Pressevertretern in Berichterstattung zum Deutschen Ärztetag

c) Aufmerksamkeitsgenerierung über Unterstützung „Unbeteiligter“

- i)Einsatz von Patientenvertretern für die Bedeutung der Zusatz-Weiterbildung

- ii)Einschalten von Politik zu ggf. unintransparenten Vorgängen

Aufgrund der Knappheit der Zeit wurde beschlossen, alle drei Schritte parallel zu verfolgen, also Delegierte für einen Antrag auf Erhalt der Zusatzbezeichnung zu gewinnen, die Delegierten des Deutschen Ärztetages zu kontaktieren und drittens Pressearbeit zu leisten. Bereits am Freitag den 27.04.2018 wurden per Email alle Delegierten des Deutschen Ärztetags gemeinsam vom geschäftsführenden Vorstand der Deutschen Gesellschaft für Phlebologie (Prof. Mumme, Dr. Hartmann, Dr. Mendoza, Prof. Stücker), dem Vorsitzenden des Berufsverbands (Dr. Gerlach) und dem Past-Präsident der UIP Prof. Rabe angeschrieben und auf die Problematik der geplanten Abschaffung der Zusatzbezeichnung Phlebologie hingewiesen. Was waren die Argumente, warum die Zusatzweiterbildung Phlebologie unverzichtbar ist? demokratischen und in jedem Fall

1. Der interdisziplinäre Charakter der $\mathrm{Zu}$ satzweiterbildung Phlebologie, die nicht vollständig in den anderen Fächern enthalten ist, die ihre phlebologischen Inhalte fachspezifisch definieren.

2. Die Versorgungssituation: Ca. $30 \%$ der Bevölkerung sind von Venenleiden betroffen. Aufgrund der sich verändernden Altersstruktur ist bis 2050 mit einem Anstieg der Prävalenz zu rechnen.

3. Die europäische Entwicklung: Erst im Herbst 2017 hat die UEMS mit dem Multiple Joint Committee Phlebology die Weichen für eine europäische interdisziplinäre Phlebologie gestellt. Dabei waren sicher auch die positiven Erfahrungen mit der Phlebologie in Deutschland ein Motor.

Sicherheitshalber wurden die Deutsche Dermatologische Gesellschaft, der Berufsverband der Dermatologen sowie die DDG und DGA über die Vorgänge informiert. Bei einer Vorstandssitzung der DGG am Samstag den 29.04. war laut Bericht von Herrn Dr. Thomas Noppeney die Einstellung so, dass eine Beibehaltung der Zusatzweiterbildung Phlebologie aussichtslos sei und die Schlacht verloren sei. Die DGA äußerte sich über ihren Vorsitzenden Prof. Dr. Sebastian Schellong in einem Telefonat mit Prof. Stücker ähnlich. Auch er hielt weitere Aktivitäten zugunsten der Phlebologie nicht für sinnvoll. Anders reagierten die Deutsche Dermatologische Gesellschaft und der Berufsverband der Dermatologen. Sie gaben unverzüglich eine Kurzexpertise zur Epidemiologie, Versorgungsbedarf und Versorgungsvolumina peripherer Venenerkrankungen in Deutschland beim Institut für Versorgungsforschung in der Dermatologie bei Pflegeberufen (IVDP, Direktor

\section{Was bedeutet eine berufsbegleitende Weiterbildung? (Beratungser- gebnis der Ständigen Konferenz „Ärztliche Weiterbildung” am 16.04.2018)}

„Eine berufsbegleitende Weiterbildung ist die Erlangung einer Zusatz-Weiterbildung neben einer hauptberuflichen ärztlichen Tätigkeit unter Anleitung zur Weiterbildung befugter Ärzte oder durch Unterweisung in anerkannten Weiterbildungskursen bzw. Fallseminaren.
Univ.-Prof. Dr. Matthias Augustin) in Auftrag. Der BVDD konnte Herrn Dr. Sebastian Biltz aus München dazu gewinnen, als Delegierter einen Antrag auf Beibehaltung der Phlebologie zu stellen. Frau Prof. Leena Bruckner-Tudermann, Präsidentin der DDG, versicherte in einem Telefonat mit Prof. Dr. Markus Stücker, dass auch die DDG in höchstem Maße interessiert an der Beibehaltung der Zusatzweiterbildung Phlebologie sei. Für die Dermatologie war die Lage insofern besonders prekär, als erst zum 01. April 2018 der „AOK-Vertrag endovenöse Leistungen in Baden-Württemberg" in Kraft getreten war (Vereinbarung zur besonderen Versorgung nach $₫ 140 \mathrm{a}$ SGB V im Bereich der ambulanten Venentherapie (endovenöse Lasertherapie, Radiofrequenzablation)). Dieser Vertrag definiert als fachliche Voraussetzungen durch den Vertragsarzt, dass es entweder ein Facharzt für Gefäßchirurgie und/oder Facharzt für Chirurgie und/oder Arzt mit der Zusatzbezeichnung Phlebologie sein müsse. Die Dermatologen können also nur über die Zusatzbezeichnung Phlebologie an diesem Vertrag teilnehmen. Die zwischen Prof. Augustin (CVderm) und Prof. Stücker abgestimmte Expertise konnte folgende Erkenntnisse zur Versorgung der Venenkrankheiten in Deutschland ableiten:

1. In Deutschland leiden mind. $22 \%$ der Bevölkerung und damit ca. 18 Mio. Personen unter abklärungs- oder behandlungsbedürftigen Venenerkrankungen.

2. Deutschlandweit werden jährlich etwa 4,1 Millionen ambulante phlebologische Behandlungen durchgeführt.

3. Stationär werden in Deutschland jährlich etwa 125.000 Fälle mit peripheren Venenerkrankungen als Hauptdiagnose dokumentiert, davon ca. 88.500 mit Varizen der unteren Extremität (ICD-10 I83) und ca. 35.300 mit Phlebitis/ Thrombophlebitis (I80)

4. Die Anzahl stationärer Behandlungen für Varizen ist in den letzten 10 Jahren um $16 \%$ zurückgegangen, in den letzten 17 Jahren um 47\%.

5. Die Anzahl und damit auch der Anteil ambulanter phlebologischer Behandlungen und Eingriffe ist in den letzten 5 Jahren jährlich um 5-10\% gestiegen. 
6. Hauptversorgende ambulante Fachgruppen sind Chirurgen und Dermatologen, im stationären Bereich Fachabteilungen für Chirurgie, Dermatologie und Angiologie sowie Venenfachkliniken.

7. Die Versorgungsqualität von Venenerkrankungen ist bei wichtigen Indikatoren nur moderat. So werden nur etwa 40\% der venösen Ulzera in Deutschland mit Kompressionstherapie behandelt. Nur ein kleinerer Teil der ärztlich und pflegerisch Versorgenden beherrscht die manuelle Anlage von Kompressionsbandagen.

8. Die Versorgungsqualität ist in spezialisierter Versorgung höher als in der Fläche.

9. Der Versorgungsbedarf wird zukünftig aufgrund der demographischen Entwicklung steigen, was jährlich einen Bedarfszuwachs von ca. 3-8\% bedingt.

10. Die Zusatz-Weiterbildung „Phlebologie" wird in Deutschland 2016 von 3.049 Ärzten geführt. Die Phlebologie ist dabei ein basisversorgendes Querschnittfach, das u.a. Allgemeinmediziner, Chirurgen, Dermatologen und Angiologen verbindet.

11. Etwa $87 \%$ der dermatologischen Praxen führen phlebologische Diagnostik und kleine Eingriffe durch, etwa 56\% weisen mind. einen Arzt mit der $\mathrm{Zu}$ satz-Weiterbildung „Phlebologie“ auf. Pro Jahr werden etwa 880 phlebologische Patienten/Praxis versorgt.

12. Zwischen den Kollegen mit und ohne phlebologischen Schwerpunkt (aller Fachrichtungen) bestehen weitreichende Kooperationen.

13. Die Zusatz-Weiterbildung „Phlebologie“ ist als Teil der qualitätsgesicherten, leitlinienkonformen Versorgung von hoher Bedeutung.

14. Die qualifizierte phlebologische Versorgung ist ökonomisch sinnvoll und hat sich in Studien auch als effizient erwiesen.

15. Im Interesse der zukünftigen Versorgungsqualität, der Versorgungseffizienz und des Anspruches von Patienten auf Zugang zu erkennbar qualifizierter Versorgung sollte die ZusatzWeiterbildung „Phlebologie“ erhalten bleiben.
Diese Kurzexpertise wurde mit einem entsprechenden Schreiben am Sonntag den 06.05.2018 an alle Delegierten des 121. Deutschen Ärztetags in Erfurt per Email versandt, unterschrieben von Frau Prof. Dr. Leena Bruckner-Tudermann, Herrn Dr. Steffen Gass, Herrn Prof. Dr. Matthias Augustin und Herrn Dr. Thomas Stavermann. Hervorgehoben wurden folgende Punkte:

1. Für die phlebologische Versorgung besteht ein hoher, künftig steigender Bedarf nach evidenzbasierter, qualifizierter Versorgung. Diese Versorgung weist eine zunehmende Spezialisierung auf, die in der Breite der Fächer nicht geleistet werden kann.

2. Die ambulante Versorgung von Venenkrankheiten ist von wachsender Bedeutung. Die versorgenden Praxen müssen für die Patienten und die Bevölkerung erkennbar sein, was nur durch die ZWB „Phlebologie“ hinreichend gewährleistet ist.

3. Qualität und Effizienz der Versorgung von Venenkrankheiten sind nach den versorgungswissenschaftlichen Daten unter Einschaltung der phlebologischen Experten höher. Die Phlebologie ist dabei das breit versorgende Querschnittfach, welches u.a. Allgemeinmediziner, Chirurgen, Dermatologen und Angiologen verbindet.

4. Das Verfahren der Beschlussfassung durch die SKO WB an die BÄK war intransparent und fand unter Ausschluss der betroffenen Gesellschaften und Verbände statt. Die berechtigten Einwände der Phlebologen wurden nicht zur Kenntnis genommen.

Am 29.04.2018, unmittelbar nach der Email aus der Bundesärztekammer wurde eine Zuordnung aller Delegierten des Deutschen Ärztetages zu Mitgliedern des Vorstands und Beirats der Deutschen Gesellschaft der Phlebologie und des Berufsverbands der Phlebologen erarbeitet und versandt mit der Bitte, die jeweilig zugeteilten Delegierten telefonisch kurzfristig zu kontaktieren. Die Ergebnisse dieser Gespräche wurden rückgemeldet und in der Delegiertenliste vermerkt. Dabei zeigte sich, dass zumindest in den Gesprächen nur wenige eindeutig ablehnende Stimmen gegen die Phlebologie registriert werden konnten.

Am Freitag den 04. Mai wurden die Delegierten des Deutschen Ärztetags von der Deutschen Venenliga, einer Patientenvereinigung von Venenerkrankten ebenfalls auf die Problematik hingewiesen. Von der Geschäftsführerin Frau Petra Hager-Häusler wurde in diesem Patientenschreiben auf folgende Punkte hingewiesen:

- Venenleiden sind eine Volkskrankheit. Ihre Behandlung gehört in die Hand eines Phlebologen!

- Fast 25 Millionen Deutsche sind von einer behandlungsbedürftigen Venenerkrankung betroffen!

- Nur mit Praxis-Zusatzbezeichnung Phlebologie finden Betroffene den Weg zum Spezialisten!

- Die Qualität der phlebologischen Versorgung ist entscheidend und kann Leben retten!

- Mindestens 40.000 Tote p.a. aufgrund tödlicher Lungenembolien, meist verursacht durch eine unerkannte Venenerkrankung mit Folgen: Thrombose Lungenembolie!

- 80.000 Betroffene mit venös bedingtem Ulcus cruris - oft mangels phlebologisch qualifizierter Therapien!

Durch entsprechende Meldungen war zwischendurch auch die Presse sensibilisiert worden. Die Ärztezeitung informierte über die geplante Abschaffung der Zusatzweiterbildung. Die Journalisten traten dabei auch mit Herrn Dr. Franz Bartmann, dem Vorsitzenden der Weiterbildungsgremien der Bundesärztekammer, der sich viele Jahre für die Novellierung der (Muster-)Weiterbildungsordnung (NWBO) engagiert hatte, in Kontakt.

Am Samstag den 05. Mai erschien ein ausführlicher ganzseitiger Bericht in der Frankfurter Rundschau zur drohenden Abschaffung der Zusatzweiterbildung Phlebologie mit Stellungnahmen von Frau PD Dr. Eva Valesky aus dem Beirat der DGP, Herrn Dr. Franz Bartmann und Prof. Dr. Markus Stücker.

Durch die Telefonate konnten mehrere Delegierte gewonnen werden, die einen Antrag auf Erhalt der Zusatzweiterbildung Phlebologie stellen wollten. Es bestand Einigkeit darüber, dass es sinnvoll sei mehre- 
re Anträge mit ähnlichem Inhalt zu stellen, um die Dringlichkeit der Angelegenheit zu verdeutlichen. Im unmittelbaren Vorfeld des 121. Deutschen Ärztetags am Montag den 07.05.2018 war Herr Dr. Horst Gerlach, Vorsitzender des Berufsverbands der Phlebologen, bereits vor Ort in Erfurt. Er konnte dort mit einigen Delegierten aus dem Bereich der Kassenärztlichen Vereinigungen sprechen, insbesondere auch mit Herrn Dr. Norbert Metke, KV Vorsitzender von Baden Württemberg, wegen der besonderen Brisanz für diese Landesärztekammer, da die Zusatzbezeichnung Phlebologie ganz wesentlicher Bestandteil des o.g. Sondervertrags mit der AOK war. Zum anderen konnte er mit Herrn Dr. HansFriedrich Spies, dem Vorsitzenden des Spi$\mathrm{Fa}$ (Spitzenverband Fachärzte Deutschlands e.V.) bezüglich des Widerspruchs diskutieren, dass die SpiFA einerseits das europäische Phlebologie-Curriculum unterstütze, andererseits aber nichts gegen die Abschaffung der Zusatzweiterbildung Phlebologie unternommen hatte.

Am Dienstag den 08.05.2018 reiste Prof. Markus Stücker zum Deutschen Ärztetag nach Erfurt, um mit Gaststatus vor Ort Gespräche führen $\mathrm{zu}$ können. Insbesondere mit den gewonnenen Delegierten Dr. Sebastian Biltz aus der LÄK Bayern, Dr. Lothar Rütz aus der LÄK Nordrhein, Wieland Dietrich aus der LÄK Nordrhein, Dr. Christoph Freiherr von Ascheraden aus der LÄK Baden-Württemberg, Dr. Andreas Gibb aus der LÄK Mecklenburg-Vorpommern und Dr. Ulrike Beiteke aus der LÄK Westfalen Lippe. Ebenfalls einen Antrag einbringen wollte Herr Dr. Lensing aus der LÄK Niedersachsen. Die Delegierten waren bereits im Vorhinein mit Argumenten und Textvorschlägen für Anträge versorgt worden. Die Feinabstimmung geschah dann im persönlichen Gespräch vor Ort. An dieser Stelle sei den antragstellenden Delegierten auch noch einmal ein herzlicher Dank ausgesprochen. Ohne ihre Aktivität hätte die Zusatzweiterbildung Phlebologie nicht gerettet werden können. War Prof. Stücker aufgrund der zahlreichen positiven Rückmeldungen aus den Telefonaten mit den Delegierten sehr optimistisch nach Erfurt gereist, musste er doch vor Ort auch kritische Stimmen zur Kenntnis nehmen. So wurde beispielsweise in der Abge- ordnetenbesprechung in Halle 2 deutlich, dass die Niedergelassenen insgesamt $\mathrm{Zu}$ satzweiterbildungen sehr kritisch gegenüber eingestellt waren und am liebsten gar keine Zusatzweiterbildungen mehr wollten. Wenn allerdings Zusatzweiterbildung, sollten diese berufsbegleitend erwerbbar sein, um so auch dem in der Praxis niedergelassenen Kollegen zur Verfügung zu stehen. Ähnliches wurde von Dr. Theo Windhorst, dem Präsidenten der LÄK Westfalen-Lippe und auch von Herrn Dr. Hans-Albert Gehle, dem Vorsitzenden des Marburger BundArbeitskreises Fort- und Weiterbildungspolitik, angemerkt, die der Zusatzweiterbildung Phlebologie zwar grundsätzlich positiv gegenüberstanden, aber die relativ geringen Zahlen der abgeschlossenen Weiterbildungen als sehr kritisch sahen und eine grundsätzliche Änderung des Weiterbildungsprinzips bei der Zusatzweiterbildung Phlebologie für sinnvoll hielten, weg von vorgeschriebenen Zeiten an definierten Weiterbildungsstätten hin zu einer kompetenzorientierten berufsbegleitend $\mathrm{zu}$ erwerbenden Zusatzweiterbildung.

Erst am letzten Tag des Deutschen Ärztetages, am Freitag den 11.05.2018, stand dann die Novellierung der MusterWeiterbildungsordnung auf der Tagesordnung. In der einführenden Rede von etwa 80 Minuten Dauer ging Herr Dr. Bartmann ungewöhnlich ausführlich auf die Zusatzweiterbildung Phlebologie ein. Er wies auf eine Kampagne der Deutschen Gesellschaft für Phlebologie hin, wie sie für die Geschichte des Deutschen Ärztetages einzigartig sei. Hielt er dies noch für gerechtfertigt, erschienen ihm die Presseaktivitäten überzogen. Insbesondere die Fragen der Journalisten, ob durch den Wegfall der $\mathrm{Zu}$ satzweiterbildung Phlebologie nicht die Versorgung gefährdet sei, erschienen ihm zu weitgehend vor dem Hintergrund der seiner Meinung nach geringen Zahlen an abgeschlossenen Prüfungen in diesem Bereich. Bereits in dieser Rede deutete er an, dass ein Ausweg aus dieser Situation seiner Meinung nach nur eine berufsbegleitende Form der Weiterbildung sein könne. In der nun folgenden Aussprache meldeten sich von etwa 50 Wortbeiträgen 5 Delegierte zugunsten der Phlebologie zu Wort. Nach den Redebeiträgen von Herrn Dr. Christoph von Ascheraden und Herrn Dr. Lo- thar Rütz ergriff Herr Dr. Bartmann noch einmal das Wort, um erneut auf die Möglichkeit der berufsbegleitenden Weiterbildung gerade für die Phlebologie hinzuweisen. Nach zwei weiteren Redebeiträgen von Herrn Dr. Gibb und Herrn Dietrich stellte Herr Dr. Bartmann in einem erneuten Redebeitrag klar, dass eine berufsbegleitende Zusatzbezeichnung keine Zusatzbezeichnung zweiter Klasse wäre und dass er nun definitiv auf einen Antrag auf berufsbegleitende Erwerbbarkeit der Phlebologie warten würde. Nach einer kurzen und intensiven Beratschlagung wurden dann durch Herrn Dr. Rütz und Herrn Dietrich sowie durch Herrn Dr. Gibb nach Rücksprache mit Prof. Stücker diese Anträge gestellt. Unter Kenntnis dieser neuen Antragssituation und unter Aufgreifen der Argumente von Herrn Dr. Bartmann bezog Frau Dr. Beiteke dann auch noch einmal Stellung zugunsten der Zusatzweiterbildung Phlebologie. Die Rednerliste wurde schließlich geschlossen und der Abstimmungsprozess begann. Durch die Vertreterinnen des zuständigen Dezernats der Bundesärztekammer, Frau Dr. Güntert und Frau Dr. Höft, wohl in Abstimmung mit Herrn Dr. Bartmann waren die Anträge zur Phlebologie zu einem Block angeordnet worden, wobei zunächst über den gemeinsamen Antrag von Dr. Rütz und Herrn Dietrich mit der Antragsnummer 81 abgestimmt werden sollte mit dem Beschlussvorschlag: „Der Erwerb der Zusatzweiterbildung Phlebologie soll berufsbegleitend möglich sein." Dieser Antrag wurde angenommen. Anschließend wurde dann erst über den Antrag auf Beibehaltung der Zusatzweiterbildung Phlebologie der Kollegen Rütz und Biltz mit der Nummer 21 abgestimmt. Die beantragte Vorstandsüberweisung wurde abgelehnt, der gesamte Antrag dann in der ausgezählten Abstimmung mit 124 zu 82 Stimmen angenommen. Das Drama hatte einen glücklichen Ausgang genommen. Die nächsten Monate müssen nun genutzt werden, die neue, moderne Form der Weiterbildungsordnung weiter zu definieren.

Abschließend sei ein Wort des Dankes gesagt. Dieser Dank gilt dem Vorstand und Beirat der Deutschen Gesellschaft für Phlebologie und dem Berufsverband der Phlebologen sowie dem Vorstand der Deutschen Dermatologischen Gesellschaft und 
dem Berufsverband der Dermatologen. Gedankt sei aber auch - trotz aller berechtigten Kritik an der unzureichenden formalen Beteiligung der Fachgesellschaften an der Entscheidung, die Zusatzweiterbildung Phlebologie nicht beizubehalten Herrn Dr. Bartmann, der schließlich durch seine Hinweise für die berufsbegleitende
Erwerbbarkeit der Phlebologie die Akzep$\tan z$ bei den Delegierten sicherlich deutlich gesteigert hat sowie den Vertreterinnen des Dezernats Aus- und Weiterbildung der Bundesärztekammer, Frau Dr. Höft und Frau Dr. Güntert, für ihre stets offene und faire Umgangsweise mit dem Vorstand der DGP.
Die Organisation der Kampagne für die Phlebologie wäre ohne den unermüdlichen Einsatz von Frau Anja Pielhau aus dem Sekretariat der Deutschen Gesellschaft für Phlebologie nicht möglich gewesen. Ihr sei an dieser Stelle besonders gedankt. 